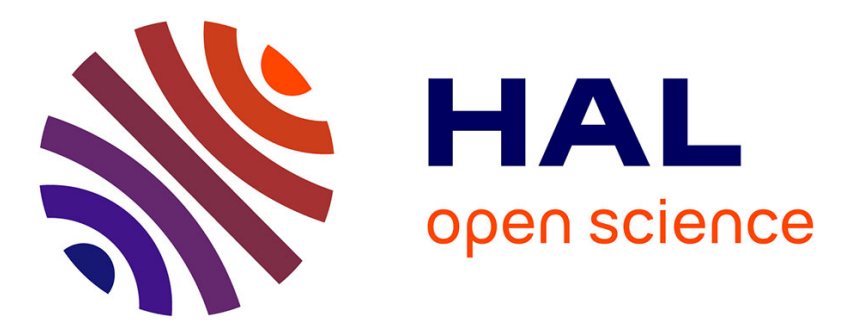

\title{
Approche méso-macro pour la simulation de la mise en forme des composites à renforts tissés
}

Philippe Boisse, Jean-Luc Daniel, Benjamin Hagege, Damien Soulat

\section{To cite this version:}

Philippe Boisse, Jean-Luc Daniel, Benjamin Hagege, Damien Soulat. Approche méso-macro pour la simulation de la mise en forme des composites à renforts tissés. Revue Européenne de Mécanique Numérique/European Journal of Computational Mechanics, 2006, 15 (1-2-3), pp.93-106. 10.3166/remn.15.93-106 . hal-00081064

\section{HAL Id: hal-00081064 https://hal.science/hal-00081064}

Submitted on 16 Mar 2018

HAL is a multi-disciplinary open access archive for the deposit and dissemination of scientific research documents, whether they are published or not. The documents may come from teaching and research institutions in France or abroad, or from public or private research centers.
L'archive ouverte pluridisciplinaire HAL, est destinée au dépôt et à la diffusion de documents scientifiques de niveau recherche, publiés ou non, émanant des établissements d'enseignement et de recherche français ou étrangers, des laboratoires publics ou privés. 


\title{
Approche meso-macro pour la simulation de la mise en forme des composites à renforts tissés
}

\author{
P. Boisse ${ }^{*}$ - J.L. Daniel** - B. Hagege ${ }^{* *}$ - D. Soulat $* *$ \\ * Laboratoire de Mécanique des Contacts et des Solides \\ LaMCoS, UMR CNRS 5514, INSA de Lyon \\ 69621 Villeurbanne Cedex \\ ** Laboratoire de Mécanique des Systèmes et des Procédés \\ LMSP, UMR CNRS 8106 ENSAM- Polytech'Orléans \\ 45072 Orléans Cedex 2 \\ Philippe.Boisse@insa-lyon.fr
}

RÉSUMÉ. Une simulation élément finis de la mise en forme des renforts tissés de composites est présentée. Les éléments finis sont composés de mailles tissées et sont basés sur une forme simplifiée du travail virtuel des efforts intérieurs prenant en compte uniquement les tensions biaxiales et le cisaillement plan. Les comportements de la maille pour ces deux grandeurs peuvent être obtenus soit par des expériences soit par des simulations à l'échelle mesoscopique. Celles-ci sont spécifiques à l'analyse des milieux fibreux. On présente la mise en forme hémisphérique d'un renfort tissé très déséquilibré. On montre que si les tensions suffisent à décrire la non symétrie, la description des plissements nécessite la prise en compte du cisaillement.

ABSTRACT A finite element made of woven unit cells under biaxial tension and in-plane shear is proposed for the simulation of fabric forming. The simulation is based on a simplified dynamic equation accounting for tension and in-plane shear strain energy. The biaxial tensile properties and the in-plane shear properties can be determined both experiments or obtained by mesoscopic $3 D$ finite element analyses of the woven unit cell. The results obtained by the simulations of a hemispherical forming process on a very unbalanced fabric are compared to experiments. It is shown that the tension strain energy permits to describe the asymmetry of the response but that the computation of wrinkles and of the deformed states when the locking angle is exceeded needs to take the in-plane shear stiffness and its evolution with shear angle into account.

MOTS-CLÉS: Renforts tissés, Composites, Simulation de mise en forme, Eléments finis, Mesomacro.

KEYWORDS: Fabrics/textiles, Composites, Forming simulation Finite element, Meso-macro. 


\section{Introduction}

Le comportement mécanique des renforts tissés utilisés dans les composites est fortement influencé par le tissage des mèches chaîne et trame. Les rigidités principales du tissé sont celles de tension. Le comportement en tension est nonlinéaire compte-tenu des liaisons et des ondulations entre les deux réseaux qui conduisent à des non-linéarités géométriques locales (Kawabata et al., 1973). Ce comportement en tension est analysé dans le présent travail par des simulations 3D de traction biaxiale de la maille élémentaire (Boisse et al, 2001). On montre en particulier que pour ces analyses, les dérivées objectives classiques (Jaumann, Green Naghdi) (Dafalias, 1983) doivent être remplacées dans les modèles hypoélastiques par des dérivées objectives construites sur la rotation des fibres (Hagege, 2004).

La rigidité de cisaillement dans le plan des renforts tissés est faible jusqu'à un angle limite. On montre par des mesures optiques à l'échelle mesoscopique lors d'un essai par cadre déformable que ceci correspond à une phase de rotation de corps rigide de chaque mèche. Une deuxième phase correspondant à un blocage et un écrasement latéral des mèches conduit à des rigidités beaucoup plus fortes. Des éléments finis spécifiques composés de mailles tissées et intégrant les résultats précédents sont présentés. On montre sur un exemple de simulation d'emboutissage d'un renfort tissé utilisé dans l'industrie automobile que la prise en compte du cisaillement est importante si l'on dépasse l'angle limite. Elle permet de décrire les plissements qui apparaissent alors. Les simulations sont comparées à un résultat expérimental dans le cas d'un emboutissage hémisphérique non-symétrique.

\section{Forme simplifiée du travail des efforts intérieurs pour les structures tissées}

Les renforts tissés considérés dans cette étude sont ceux classiquement utilisés dans les composites. Les fils sont constitués de milliers de fibres de verre, carbone ou aramide. Les fils de trame et de chaîne sont liés par les tissages classiques : taffetas, satin, sergé. Le comportement mécanique qui en résulte est très spécifique compte tenu des possibles mouvements relatifs des fils et des fibres.

Pour simuler les déformations et en particulier la mise en forme de ces renforts tissés différentes approches sont possibles. Le tissé peut être considéré comme un milieu continu anisotrope et le modèle macroscopique doit traduire les spécificités liées à la structure interne (Rogers, 1989)(Spencer, 2000). Si ces approches permettent une implémentation directe dans les codes éléments finis classiques, l'actualisation des caractéristiques au cours de la déformation est difficile et certains aspects ne sont en général pas intégrés (interactions, blocage...). A l'opposé d'autres approches sont basées sur une discrétisation complète de la structure interne du tissé (Ben Boubaker et al, 2002). L'effort numérique pour la structure complète est alors très important et contraint à beaucoup simplifier le modèle local. La méthode présentée associe une discrétisation éléments finis à une analyse mesoscopique de la maille élémentaire tissée. Le comportement mécanique de la 
maille élémentaire (échelle mésoscopique) est analysé à la fois par des analyses numériques et expérimentales.

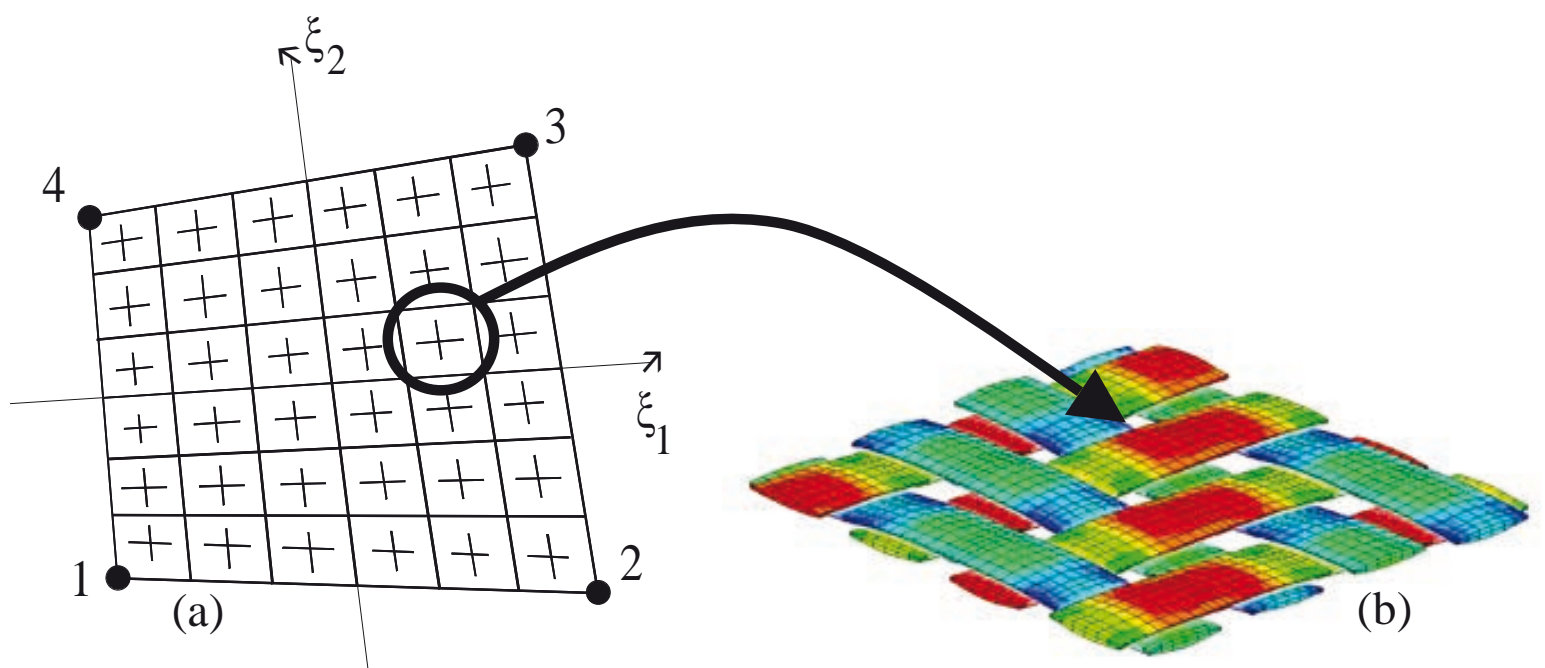

Figure 1. Elément fini composé de mailles tissées (a) et simulation d'un sergé $2 \times 2$ en tension biaxiale (b)

Toutes les rigidités sont faibles par rapport à celles de tension dans la direction des mèches compte tenu des mouvements relatifs possibles entre les mèches et les fibres. Une approche efficace doit prendre en compte ces spécificités et être aussi simple que possible tout en traduisant les aspects principaux du comportement mécanique des renforts tissés. En particulier

- Le comportement non-linéaire dû aux ondulations des mèches

- Le verrouillage en cisaillement et les comportements différents avant et après le verrouillage.

Dans l'approche proposée, on considère une forme simplifiée du travail virtuel des efforts intérieur sur une maille élémentaire tissée :

$$
\mathrm{T}_{\text {int }}^{\text {cell }}(\eta)=\varepsilon_{11}(\eta) \mathrm{T}^{11} \mathrm{~L}_{1}+\varepsilon_{22}(\eta) \mathrm{T}^{22} \mathrm{~L}_{2}+\gamma(\eta) \mathrm{C}
$$

$\mathrm{L}_{1}, \mathrm{~L}_{2}$ sont les dimensions de la maille. C est le couple créé par le cisaillement dans le plan $\gamma$ sur la maille p. $\mathrm{T}^{11}$ et $\mathrm{T}^{22}$ sont les composantes de tension dans les directions des mèches chaîne et trame. Cette forme demande la connaissance des tensions en fonction des déformations axiales $\mathrm{T}^{11}\left(\varepsilon_{11}, \varepsilon_{22}\right)$ et $\mathrm{T}^{22}\left(\varepsilon_{11}, \varepsilon_{22}\right)$. Ces surfaces de tensions permettent de prendre en compte l'effet de l'embuvage et de l'entrelacement des mèches chaîne et trame. Le couple $C$ doit dépendre de $\gamma$ de manière à différencier le comportement avant et après le blocage. Ces quantités peuvent être déterminées par différents moyens simples ou sophistiqués, expérimentaux ou numériques.

\section{Analyse mesoscopique du comportement mécanique de la maille tissée}

\subsection{Comportement en tension biaxiale}

Les tests de tensions sur des renforts tissés montrent une forte non-linéarité de comportement suivie d'une zone linéaire (Buet et al, 2001). Cette non-linéarité est 
liée au changement de géométrie à l'échelle de la maille. Le tissage amène une ondulation des mèches du tissu. Sous tension, la mèche tend à devenir droite, ce qui modifie l'ondulation dans la direction de la tension, mais également dans la direction transverse. Ce phénomène est amplifié par l'écrasement des mèches. C'est un phénomène biaxial compte tenu du tissage. La détermination des surfaces de tension par des analyses éléments finis 3D sont intéressantes car elles évitent des expériences difficiles et peuvent être réalisées sur des renforts tissés avant leur réalisation (Boisse et al 2001). Elles fournissent aussi des grandeurs locales très difficiles à mesurer (figure 1b).

Néanmoins l'analyse éléments finis du comportement de la maille élémentaire n'est pas classique car chaque mèche est composée de quelques milliers de fibres et leur comportement et très différent de celui d'un milieu continu classique. La difficulté principale consiste à traduire ce comportement en modélisant la maille élémentaire avec des éléments 3D. Deux points sont particulièrement importants. Le premier consiste à suivre strictement la direction des fibres au cours de la transformation pour écrire le comportement anisotrope. Les approches hypoélastiques classiquement utilisées dans les logiciels industriels (tel que Abaqus) actualisent les directions d'orthotropie par la rotation propre (Green-Nagdhi) ou la rotation du repère corotationnel (Jaumann). Ceci n'est pas satisfaisant pour un milieu fibreux. Une dérivée objective basée sur la rotation de la fibre a pour cela été construite et implémentée dans Abaqus (Hagege, 2004).

Le deuxième point important concerne la description de la loi d'écrasement transverse. Compte tenu des ondulations, les tensions dans les mèches créent des écrasements transverses importants (classiquement jusqu'à 50\%) compte tenu de la très faible rigidité initiale dans cette direction. La loi de comportement en écrasement transverse joue donc un rôle majeur (Baoxing et al, 1999).

\subsection{Comportement en cisaillement transverse}
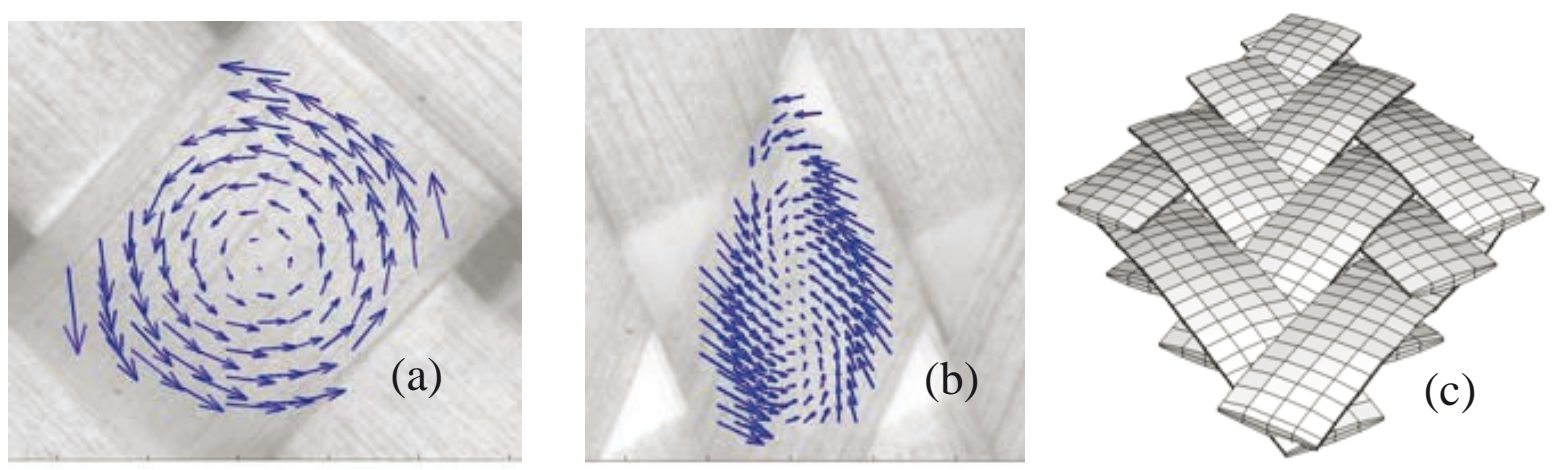

Figure 2. Champ de déplacement dans la mèche avant (a) et après (b )le blocage. (c) Analyse par élément finis 3D

Les études concernant le cisaillement plan des tissés sont nombreuses probablement parce qu'il s'agit du mode principal de déformation (Prodromou et al, 1997) (McGuinness et al, 1998). Les analyses expérimentales utilisant un cadre déformable classique montrent que l'on doit distinguer deux zones principales dans le comportement en cisaillement des renforts tissés (figure 3). Des mesures des 
champs de déplacements par corrélation d'images (Vacher et al, 1999) permettent de distinguer une première partie (pour les angles inférieurs à l'angle dit de blocage) correspondant à la rotation des mèches sans déformation (figure $2 a$ ) où les couples sur les cellules tissées sont très faibles (figure 3). Ensuite un contact latéral entre les mèches conduit à un blocage et une grande augmentation de l'effort de cisaillement (figure 2b)(Boisse et al, 2005). La figure 3 montre que les mesures optiques de la déformation diffèrent sensiblement de la mesure de variation d'angle du cadre car elles permettent de s'affranchir des problèmes de liaison au bord de l'échantillon.

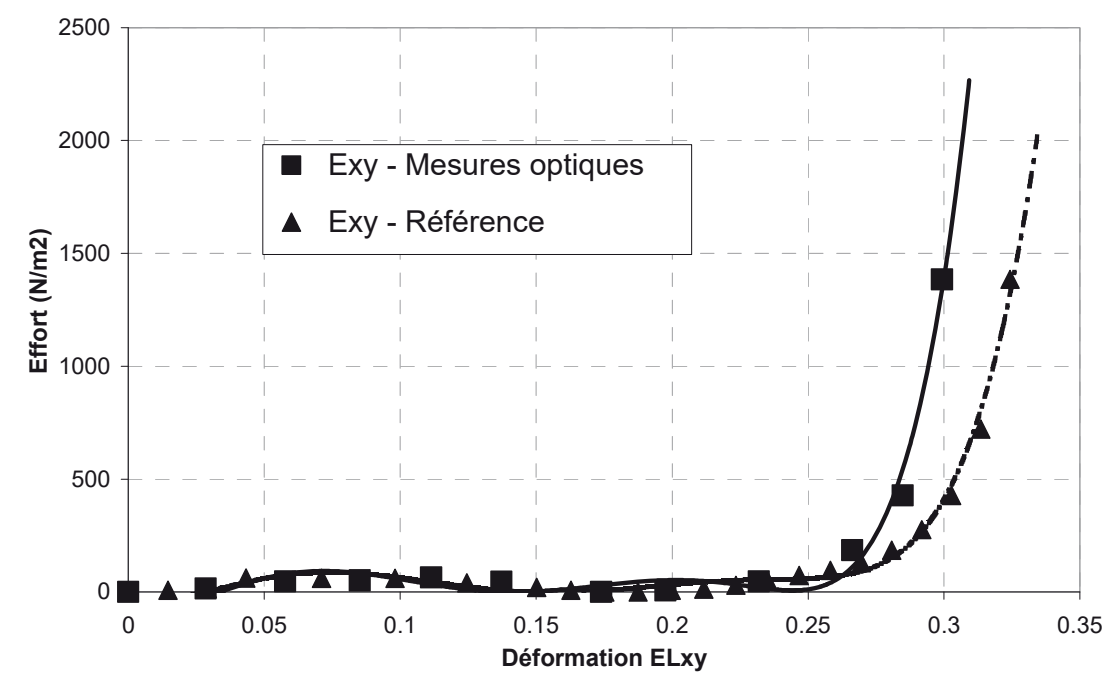

Figure 3. Courbe d'effort dû au cisaillement plan

\section{Eléments finis de tissés}

Des éléments finis sont construits à partir de la forme simplifiée du travail virtuel des efforts intérieurs [1]. On obtient une forme explicite des efforts nodaux intérieurs. La quadrature dépend du nombre de mèches par élément. Si $\mathrm{n}_{\mathrm{c}}$ et $\mathrm{n}_{\mathrm{t}}$ sont les nombres de fils dans les directions chaîne et trame, on calcule les efforts intérieurs à partir des quatre positions:

$$
\lambda_{1}=(-1)^{\gamma}\left(\left(n_{c}^{2}-1\right)\left(3 n_{c}^{2}\right)^{-1}\right)^{1 / 2} \quad \lambda_{2}=(-1)^{\delta}\left(\left(n_{t}^{2}-1\right)\left(3 n_{t}^{2}\right)^{-1}\right)^{1 / 2}
$$

$\gamma$ et $\delta$ valent 1 ou 2 . Les efforts intérieurs sont alors obtenus directement par:

$$
\left(\mathrm{F}_{\mathrm{int}}^{\mathrm{e}}\right)_{\mathrm{s}}=\sum_{\gamma=1}^{2} \sum_{\delta=1}^{2} \frac{\mathrm{n}_{\mathrm{c}} \mathrm{n}_{\mathrm{t}}}{4}\left(\begin{array}{l}
\mathrm{B}_{11 \mathrm{~s}}\left(\lambda_{1}, \lambda_{2}\right) \mathrm{L}_{1} \mathrm{~T}^{11}\left(\lambda_{1}, \lambda_{2}\right)\left\|\mathrm{g}_{1}\left(\lambda_{1}, \lambda_{2}\right)\right\|^{-2} \\
+\mathrm{B}_{22 \mathrm{~s}}\left(\lambda_{1}, \lambda_{2}\right) \mathrm{L}_{2} \mathrm{~T}^{22}\left(\lambda_{1}, \lambda_{2}\right)\left\|\mathrm{g}_{2}\left(\lambda_{1}, \lambda_{2}\right)\right\|^{-2}+\mathrm{B}_{\gamma \mathrm{s}}\left(\lambda_{1}, \lambda_{2}\right) \mathrm{C}\left(\lambda_{1}, \lambda_{2}\right)
\end{array}\right)
$$

\section{Simulation d'une mise en forme d'un tissu très déséquilibré}

On montre sur l'exemple de simulation d'emboutissage d'un renfort tissé utilisé dans l'industrie automobile présenté figure 4 que la prise en compte du cisaillement est importante si l'on dépasse l'angle limite. Elle permet de décrire 
les plissements qui apparaissent alors. Les simulations avec et sans prise en compte du cisaillement sont comparées figure 4 au résultat expérimental dans le cas d'un emboutissage hémisphérique non-symétrique.
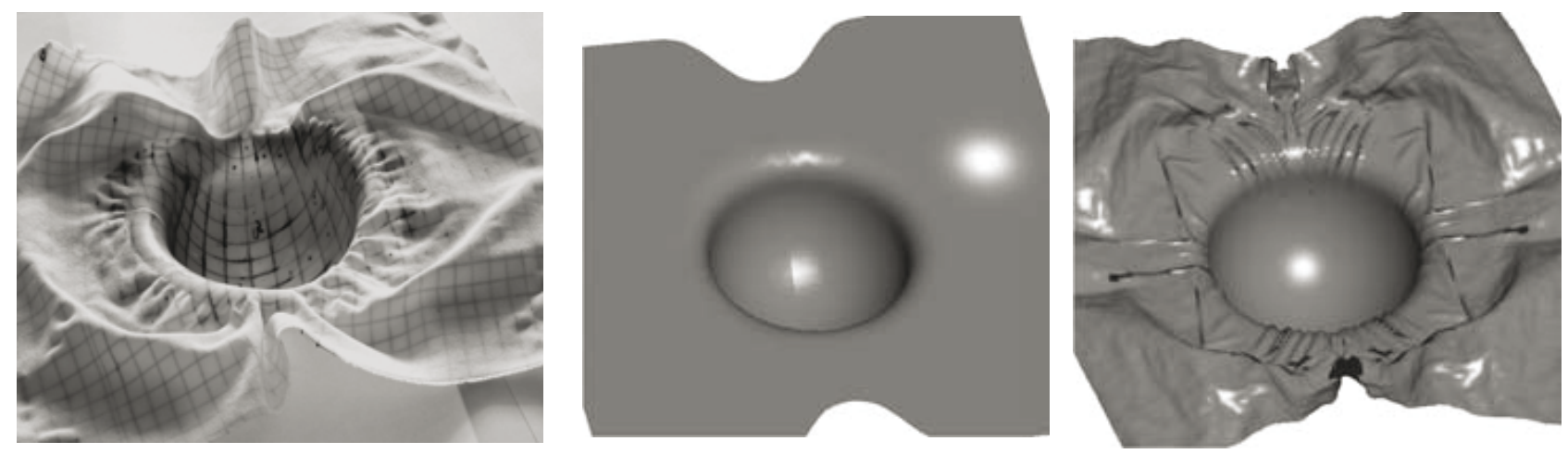

Figure 4. Emboutissage hémisphérique d’un tissu très déséquilibré

\section{Bibliographie}

Baoxing C., Chou T.W. "Compaction of woven-fabric preforms in liquid composite molding processes : single layer deformation", Composite Science and Technology, 59, 1999, p. 1519-1526.

Ben Boubaker B., Haussy B., Ganghohher J.F., "Discrete models of woven structures drapping and stability analysis", CRAS Paris, Série mécanique, 330, 2002, p 871-877

Boisse P., Gasser A., and Hivet G., "Analyses of fabric behaviour : determination of the biaxial tension-strain surfaces and their use in forming simulations". Composites Part A., 32-10, 2001, p. 1395-1414

Boisse P., Zouari B., Gasser A., "A mesoscopic approach for the simulation of woven fibre composite forming", Composites Science and Technology ,65, 2005, p.429-436

Buet K., Boisse Ph., "Experimental analysis and models for biaxial mechanicalbehaviour of composite woven reinforcements", Experimental Mech., 41, 3, 2001, p. 260-269

Dafalias Y.F., "Corotational rates for kinematic hardening at large plastic deformations", Trans. of the ASME, J. of Ap. Mech., 50,1983, p. 561-565

Hagège B., Simulation du comportement mécanique des milieux fibreux en grandes transformations : application aux renforts tricotés, Thèse de doctorat, ENSAM Paris, 2004.

Kawabata S., Niwa M., and Kawai H. "The Finite Deformation Theory of Plain Weave Fabrics Part I: The Biaxial Deformation Theory", J. Textile Inst., 64, 1, 1973, p. 21-46.

McGuinness G. B., Bradaigh C. M. O., "Characterisation of thermoplastic composite melts in rhombus-shear: the picture-frame experiment", Composites Part A, 1998, 29,1-2, p. 115132.

Prodromou, A.G. and J. Chen, "On the relationship between shear angle and wrinkling of textile composite performs", Composites Part A, 28, 5, 1997, p. 491-503.

A.J.M Spencer. Theory of fabric-reinforced viscous fluid. Composites Part A, 31, 2000, p. 1311-1321.

Rogers. T.G. Rheological characterisation of anisotropic materials, Composites, 20, 1989, p. 21-27.

Vacher P.,Dumoulin S., Arrieux R., "Determination of the forming limit diagram from local measurement using digital image analysis", International Journal of Forming Processes, 2-3-4,1999, p. 395-408. 